\title{
A phase field model for the mixture of two incompressible fluids and its approximation by a Fourier-spectral method
}

\author{
Chun Liu ${ }^{\mathrm{a}, *}$, Jie Shen ${ }^{\mathrm{b}}$ \\ a Department of Mathematics, Pennsylvania State University, University Park, PA 16802, USA \\ ${ }^{\mathrm{b}}$ Department of Mathematics, Purdue University, West Lafayette, IN 47907, USA
}

Received 27 April 2002; received in revised form 17 January 2003; accepted 20 January 2003

Communicated by R. Temam

\begin{abstract}
A phase field model for the mixture of two incompressible fluids is presented in this paper. The model is based on an energetic variational formulation. It consists of a Navier-Stokes system (linear momentum equation) coupled with a Cahn-Hilliard equation (phase field equation) through an extra stress term and the transport term. The extra stress represents the (phase induced) capillary effect for the mixture due to the surface tension. A Fourier-spectral method for the numerical approximation of this system is proposed and analyzed. Numerical results illustrating the robustness and versatility of the model are presented. () 2003 Elsevier Science B.V. All rights reserved.
\end{abstract}

Keywords: Navier-Stokes equations; Cahn-Hilliard equation; Mixture; Fourier-spectral method

\section{Introduction}

The hydrodynamics of mixture of different materials play an increasingly important role in many current scientific and engineering applications. Among them, the interfacial dynamics is one of the fundamental issues in hydrodynamics and rheology $[18,30,37,52]$ of these materials. Conventionally, the model for the mixture consists of separate hydrodynamic system of each component, together with the free interface that separates them. From another point of view, the mixture can be treated as a special type of non-Newtonian fluids. The final rheology property reflects the competition between the kinetic energy and the "elastic" mixing energy $[8,18]$.

The interfacial dynamics in the mixture of different fluids, solids or gas have attracted attentions for more than two centuries. Many surface properties, such as capillarity, are associated with the surface tension through special boundary conditions on the interfaces $[18,30,37,52]$.

In classical approaches, the interface is usually considered to be a free surface that evolves in time with the fluid (the kinematic boundary condition). The dynamics of the interface at each time is determined by the following stress (force) balance condition:

$$
[T] \cdot n=m H n,
$$

\footnotetext{
* Corresponding author.

E-mail addresses: liu@math.psu.edu (C. Liu), shen@math.purdue.edu (J. Shen).
} 
where $[T]=[v D(u)-p I]$ is the jump of the stress across the interface $\Gamma_{t}, n$ its normal, $D(u)=\frac{1}{2}\left(\nabla u+(\nabla u)^{\mathrm{T}}\right)$ the stretching tensor, $H$ the mean curvature of the surface and $m$ the surface tension constant. This is the usual Young-Laplace junction condition (see, for instance [6,30,37,52]). The hydrodynamic system describing the mixture of two Newtonian fluids with a free interface will be the usual Navier-Stokes equations in each of the fluid domains (possibly with different density and viscosity) together with the kinematic and force balance (traction free) boundary conditions on the interface. The weak form of such a system when the density $\rho$ and viscosity $v$ may vary in the mixture can be represented exactly in the following form [44]:

$$
\int_{0}^{T} \int_{\Omega}\left[-\rho u v_{t}-\rho u u \cdot \nabla v+v \nabla u \nabla v-p \nabla \cdot v\right] \mathrm{d} x \mathrm{~d} t=\int_{0}^{T} \int_{\Gamma_{t}} m H n \cdot v \mathrm{~d} s \mathrm{~d} t
$$

for any test function $v$.

From the statistical (phase field approach) point of view, the interface represents a continuous, but steep change of the properties (density, viscosity, etc.) of two fluids. Within this "thin" transition region, the fluid is mixed and has to store certain amount of "mixing energy". This has been studied as early as 19th century by Rayleigh and van der Waals (see the wonderful survey paper by Anderson et al. [4] in this area). Such an approach coincides with the usual phase field models that were developed in the theory of phase transition (see $[16,17,49,50,63]$ and the references therein), and attracted many interests in the mathematical community (cf. $[2,15,21,55,60])$. These models allow topological changes of the interface [47] and have many advantages in numerical simulations of the interfacial motion (cf. [19]). In recent years, many researchers have employed the phase field approach in various fluid environments (cf. [3,9-11,25,28,33,36,46,48,53]).

In this paper, we study a phase field model for the mixture of two incompressible fluids. The model is based on an energetic variational formulation. In the next section, we introduce the model and present essential physical considerations leading to this model. There is a clear similarity between this system and the liquid crystal flows considered in the previous work $[27,46]$. In Section 3, we present some mathematical results concerning the limiting behaviors of the model based on the Allen-Cahn phase equation [44] and illustrate their relevance for the present model which is based on the Chan-Hilliard phase equation. In Section 4, we propose and analyze a semi-discrete Fourier-spectral method for the numerical approximation of this phase-field model. Finally in Section 5, we present numerical results obtained by using a semi-implicit time discretization scheme of the Fourier-spectral method. Our numerical examples exhibit the robustness and versatility of this phase-field approach for modeling the mixture of two incompressible fluids.

\section{A phase field model for the mixture of two incompressible fluids}

We consider the following system modeling a specific type of mixture of two incompressible fluids with same density (which is taken to be 1) and same viscosity constants (cf. [46]):

$$
\begin{aligned}
& u_{t}+(u \cdot \nabla) u+\nabla p-v \operatorname{div} D(u)+\lambda \nabla \cdot(\nabla \phi \otimes \nabla \phi)=g(x), \\
& \nabla \cdot u=0, \\
& \phi_{t}+(u \cdot \nabla) \phi=-\gamma \Delta(\Delta \phi-f(\phi))
\end{aligned}
$$

with initial conditions

$$
\left.u\right|_{t=0}=u_{0},\left.\quad \phi\right|_{t=0}=\phi_{0}
$$

and appropriate boundary conditions. 
In the above system, $u$ represents the velocity vector of the fluids, $p$ is the pressure, and $\phi$ represents the "phase" of the molecules. $\Omega \subset \mathfrak{R}^{n}$ is a bounded domain (unless otherwise stated), $v$ is the viscosity constant, $f(\phi)$ is a polynomial of $\phi$ such that $f(\phi)=F^{\prime}(\phi)$, where $F(\phi)=\left(|\phi|^{2}-1\right)^{2} / 4 \eta^{2}$ is the bulk part of the mixing energy with $\eta$ as the capillary width (width of the mixing layer), $g(x)$ is the external body force. The term $\nabla \phi \otimes \nabla \phi$ is the usual tensor product, i.e. $(\nabla \phi \otimes \nabla \phi)_{i j}=\nabla_{i} \phi \nabla_{j} \phi$. Finally, $\lambda$ corresponds to the surface tension [46] and $\gamma$ represents the elastic relaxation time of the system.

The above system models the mixture of two fluids which have the same density and viscosity. Such an approach can also be extended to the variable density and variable viscosity cases [46], even to the case of inhomogeneous surface tension, for instance, the Marangoni-Bénard convection [22,30,37,45,52] and the case involving more complicated fluids [54].

In the above system, Eq. (2.1) is the linear momentum equation, where the induced elastic stress $\nabla \phi \otimes \nabla \phi$ is due to the mixing of the different species. From this, we see that $\Delta \phi \nabla \phi=\nabla \cdot(\nabla \phi \otimes \nabla \phi)-\frac{1}{2}|\nabla \phi|^{2}$ gives the corresponding elastic force. Eq. (2.2) implies the incompressibility of both fluids in the mixture. Eq. (2.3) is the phase equation: the left-hand side of the equation represents the transport property of the phase function (that the material point does not change type, at least in the limit case); the right-hand side describes the special dissipative mechanism to the system. The choice of the Chan-Hilliard system over other systems (e.g., the Allen-Cahn system) is made to preserve the integral of $\phi$ (the volume fraction in the dynamics). It also provides a specific type of dissipative mechanism in the energy law (see Eq. (2.11)).

In $[44,46]$, we studied the system with the Allen-Cahn type of phase field equation instead of the Cahn-Hilliard type that is studied here. We proved that when $\gamma$ is small and $\lambda \sim$ surface tension $\times$ capillary width, the phase equation will approach, as $\gamma, \eta \rightarrow 0$, to the following transport equation:

$$
\phi_{t}+u \cdot \nabla \phi=0
$$

On the interface $\Gamma=\{x \in \Omega \mid \phi(x, \cdot)=0\}$ (which implies the kinematic boundary condition), the nonlinear elastic force in the momentum equation becomes

$$
\int_{\Omega} \lambda \Delta \phi \nabla \phi \cdot w \sim \int_{\Omega} \lambda|\nabla \phi|^{2} H w \cdot \frac{\nabla \phi}{|\nabla \phi|} \sim \int_{\Gamma} \text { surface tension } \cdot H w \cdot n .
$$

This gives the kinetic jump condition for two immiscible fluids: $[T] n=$ surface tension $\cdot H n$. We combined the local existence of Hamilton (cf. [34,35]) and Denisova and Solonnikov [24], the convergence method of [31] and the energy estimate to show that for fixed $\gamma$, as $\eta$ approaches zero, the model converges to an auxiliary system that is the same as the level set formulation [19,51], see Theorem 3.3 in Section 3.

In the remainder of this section, we present essential physical and mathematical arguments leading to the system (2.1)-(2.3).

\subsection{Cahn-Hilliard phase field model}

For a phase function $\phi$, assuming that the elastic (mixing) energy is given by

$$
W(\phi, \nabla \phi)=\int_{\Omega}\left\{\frac{1}{2}|\nabla \phi|^{2}+F(\phi)\right\} \mathrm{d} x,
$$

then the Cahn-Hilliard equation takes the form

$$
\phi_{t}=\nabla \cdot\left(\gamma \nabla \frac{\delta W}{\delta \phi}\right)=-\gamma \Delta(\Delta \phi-f(\phi)) .
$$


Here $\delta W / \delta \phi$ represents the variation of the energy $W$ with respect to $\phi$. Also, $f(\phi)=F^{\prime}(\phi)$. The constant $\gamma$ represents the elastic relaxation time.

The solution of (2.8) satisfies the following energy law:

$$
\frac{\mathrm{d}}{\mathrm{d} t} \int_{\Omega}\left\{\frac{1}{2}|\nabla \phi|^{2}+F(\phi)\right\} \mathrm{d} x=-\int_{\Omega} \gamma\left|\nabla \frac{\delta W}{\delta \phi}\right|^{2} \mathrm{~d} x=-\int_{\Omega} \gamma|\nabla(\Delta \phi-f(\phi))|^{2} \mathrm{~d} x .
$$

This energy dissipation relation shows the variational nature of the Cahn-Hilliard equation. In fact, the Cahn-Hilliard equation can be viewed as the gradient flow of the elastic energy $W$ in the Sobolev space $H^{-1}$, instead of the $H^{1}$ space in the case of Allen-Cahn equation. Another important feature of (2.8) is that

$$
\frac{\mathrm{d}}{\mathrm{d} t} \int_{\Omega} \phi \mathrm{d} x=0
$$

Hence, the (volume) fraction is conserved for all times [17].

It was shown that if $\gamma=\eta \epsilon$ and the bulk energy takes the usual double-well form $F(\phi)=\left(1 / 4 \eta^{2}\right)\left(\phi^{2}-1\right)^{2}$, the dynamics of Cahn-Hilliard equation (2.8) will converge, as $\eta$ approaches zero, to the dynamics of a Hele-Shaw type flow [2].

We point out that there are many physical interpretation to the Cahn-Hilliard equation (cf. $[17,63,64])$ and the Allen-Cahn equation [16]. However, in this paper, we only treat them as phenomenological equations, representing certain dynamics of the elastic properties of the materials. From the energetic point of view (see the energy law (2.11)), this choice determines the special dissipative mechanism of the system.

\subsection{Energy laws and least action principle}

The system (2.1)-(2.3) is a dissipative system. Indeed, multiplying (2.1) by $u$ and (2.3) by $\delta W / \delta \phi$, integrating by parts and summing up the results, we obtain:

$$
\frac{\mathrm{d}}{\mathrm{d} t} \int_{\Omega}\left\{\frac{1}{2}|u|^{2}+\frac{\lambda}{2}|\nabla \phi|^{2}+\lambda F(\phi)\right\} \mathrm{d} x=-\int_{\Omega}\left\{v|\nabla u|^{2}+\gamma \lambda|\nabla(\Delta \phi-f(\phi))|^{2}\right\} \mathrm{d} x .
$$

It is important to notice that the energy contributions from the induced stress term and the transport term cancel each other. This is due to the following least action principle that is hidden behind the original system. In turn, the whole coupled system can be viewed as an energetic variational formulation, which includes two different variational procedures - the gradient flow for the phase variable and the least action principle for the flow map.

We consider the action function

$$
A(x)=\int_{0}^{T} \int_{\Omega_{0}}\left\{\frac{1}{2}\left|x_{t}(X, t)\right|^{2}-\frac{\lambda}{2}\left|\nabla_{x} \phi(x(X, t), t)\right|^{2}-\lambda F(\phi(x(X, t), t))\right\} \mathrm{d} X \mathrm{~d} t .
$$

Here we can view $X$ as the Lagrangian (initial) material coordinate and $x(X, t)$ the Eulerian (reference) coordinate. $\Omega_{0}$ is the initial domain occupied by the fluids. The notation $\phi(x(X, t), t)$ indicates that $\phi$ is transported by the flow field.

For incompressible materials, we look at the volume preserving flow map $x(X, t)$ such that

$$
x_{t}(X, t)=v(x(X, t), t), \quad x(X, 0)=X .
$$

The least action principle states that the linear momentum equation (force balance) shall be the least action state, without the viscosity terms. Suppose that we have a one parameter family of such maps $x^{\eta}$ such that

$$
x^{0}=x, \quad \frac{\mathrm{d} x^{\eta}}{\mathrm{d} \eta}=y
$$


for any $y$ such that $\nabla_{x} \cdot y=0$. This is a direct consequence of the fact that the Jacobian determinant of $\partial x / \partial X$ is one. If we compute the variation of $A\left(x^{\eta}\right)=A\left(\phi\left(x^{\eta}, t\right)\right)$ with respect to $\eta$ and evaluate at $\eta=0$, the kinetic part $\frac{1}{2}\left|x_{t}(X, t)\right|^{2}$ will give the usual Euler equation part in the momentum equation, while the part due to the elastic energy leads to

$$
\begin{aligned}
& \left.\frac{\mathrm{d}}{\mathrm{d} \eta}\right|_{\eta=0} \int_{0}^{T} \int_{\Omega_{0}}\left\{\frac{\lambda}{2}\left|\nabla_{x^{\eta}} \phi\left(x^{\eta}(X, t), t\right)\right|^{2}+\lambda F\left(\phi\left(x^{\eta}(X, t), t\right)\right)\right\} \mathrm{d} X \mathrm{~d} t \\
& \quad=\int_{0}^{T} \int_{\Omega_{0}}\left\{\left.\lambda \nabla_{x}^{i} \phi \frac{\mathrm{d}}{\mathrm{d} \eta}\right|_{\eta=0} \nabla_{x^{\eta}}^{i} \phi\left(x^{\eta}, t\right)+\lambda F^{\prime}(\phi) \nabla_{x}^{j} \phi y^{j}\right\} \mathrm{d} X \mathrm{~d} t \\
& =\int_{0}^{T} \int_{\Omega_{0}}\left\{\left.\lambda \nabla_{x}^{i} \phi \frac{\mathrm{d}}{\mathrm{d} \eta}\right|_{\eta=0}\left(\nabla_{x}^{j} \phi\left(x^{\eta}, t\right) \nabla_{x^{\eta}}^{i} x^{j}\right)+\lambda F^{\prime}(\phi) \nabla_{x}^{j} \phi y^{j}\right\} \mathrm{d} X \mathrm{~d} t \\
& =\int_{0}^{T} \int_{\Omega_{0}}\left\{\lambda \nabla_{x}^{i} \phi \nabla_{x}^{j} \nabla_{x}^{i} \phi(x, t) y^{j}-\lambda \nabla_{x}^{i} \phi(x, t) \nabla_{x}^{j} \phi(x, t) \nabla_{x}^{i} y^{j}+\lambda F^{\prime}(\phi) \nabla_{x}^{j} \phi y^{j}\right\} \mathrm{d} X \mathrm{~d} t .
\end{aligned}
$$

Here, we have used the fact that $\nabla_{x} \eta x$ is the inverse matrix of $\nabla_{x} x^{\eta}$. Since $y$ is an arbitrary divergence free vector field, an integration by parts leads to the following equation:

$$
u_{t}+(u \cdot \nabla) u+\nabla p+\lambda \nabla \cdot(\nabla \phi \otimes \nabla \phi)=0,
$$

where all the pure gradient terms are absorbed in the pressure.

We point out that similar derivations were also used for the Ericksen-Leslie system $[42,43]$ in which case the elastic energy is due to the molecular orientation [23]. This is also equivalent to the principle of virtual work in the physics and chemical engineering literature $[7,23,26]$.

\subsection{Hydrodynamic equilibrium}

The existence of the hydrodynamic equilibrium states for the system (2.1)-(2.3) (the static solution with the velocity $u=0$ ) is due to the energetic variational formulation, in particular, it can be viewed as a special relation between the solution of the Euler-Lagrange equation of the elastic energy and the solution of the equation from variation of the domain to such an energy.

The least action principle (variation on the flow maps) and the fastest decent dynamics or other types of gradient flows (variation on the phase variables) come from different physics laws. In the static case, the first one is equivalent to the variation with respect to the domain and the second one is the variation of the same functional with respect to the function. It is clear that if the solutions are smooth (or regular enough), they are equivalent. Formally, the existence of the hydrodynamic equilibrium states is due to the following theorem (see, e.g. [43]):

Theorem 2.1. Given an energy functional $W(\phi, \nabla \phi)$, all solutions of the Euler-Lagrangian equation:

$$
-\nabla \cdot \frac{\partial W}{\partial \nabla \phi}+\frac{\partial W}{\nabla \phi}=0
$$

also satisfy the equation

$$
\nabla \cdot\left(\frac{\partial W}{\partial \nabla \phi} \otimes \nabla \phi-W I\right)=0 .
$$

This result shows the connection between the diffusion from the gradient flow (variation of the elastic energy with respect to $\phi$ ) and the capillary force (variation of the elastic energy with respect to the flow map, the domain in 
the static case) through Legendre transform as in $[1,5]$. This theorem guarantees the existence of the hydrodynamic equilibrium states for our system. It also gives the stability results [42] and shows that all solutions of the system (2.1)-(2.4) will approach to an equilibrium state as $t \rightarrow+\infty$. One can also derive from Theorem 2.1 the usual Pohozaev identity [62] by writing Eqs. (2.16) and (2.17) in weak forms.

In the general case, the weak solution of the Euler-Lagrange equation (due to the variation with respect to the function) may not satisfy the equation from variation of the domain. Hence, the latter equation can be treated as a regularity choice mechanism for the weak solution of the Euler-Lagrange equation [43]. This is analogous to the "stationary weak solution" in the theory of harmonic maps [56,57]. There, the variable is a vector from the domain to a ball $\phi: \Omega \rightarrow S^{n}$ and $W(\phi)=\frac{1}{2}|\nabla \phi|^{2}$. Then, (2.17) defines the "stationary weak solution" of (2.16) (cf. [56]), and ensures the monotonicity of the normalized energy of the solution. This is a very important property pertaining to the regularity of the solution and the structure of its singularities.

\subsection{Phase induced capillary effects}

For simplicity, let us look at the following well-known functional of Ginzburg-Landau type (with double-well in the bulk potential)

$$
\tilde{W}(\phi, \nabla \phi)=\int_{\Omega}\left\{\frac{\eta}{2}|\nabla \phi|^{2}+\frac{1}{4 \eta}\left(\phi^{2}-1\right)^{2}\right\} \mathrm{d} x .
$$

The part of bulk energy represents the interaction of different volume fractions of individual species (to certain degree, this corresponds to the Flory-Higgins free energy [29,39]). The gradient part is the regularization (relaxation) part. This relaxation links the mass average of the energy (especially the kinetic energy) with the volume average of the elastic energy. The gradient part is also the approximation of the interface surface energy (the surface area in this case). Since the surface tension can be derived through the variation of the surface energy [40], it is not surprising that it is the contribution of this term in the momentum equation that gives the surface tension in the limit.

Assuming that the dissipation effect is described through the following gradient flow (fastest decent) mechanism

$$
\phi_{t}+v \cdot \phi=-\frac{\gamma}{\eta} \frac{\delta \tilde{W}}{\delta \phi}=\frac{\gamma}{\eta}\left(\eta \Delta \phi-\frac{1}{\eta}\left(\phi^{2}-1\right) \phi\right)
$$

the (internal) dissipation mechanism will disappear as $\gamma$ approaches zero. Thus, the choice of the right-hand side of (2.3) is not important when $\gamma$ is small. This is verified in our numerical experiments (see Example 2 in Section 5). However, a rigorous proof of this statement is not yet available.

The constant $\eta$ in (2.18) is the capillary width of the mixture [12,13] and [55] (the width of the mixing layer). As the constant $\eta$ approaches zero, $\phi$ will approach 1 and -1 almost everywhere, and the contribution due to the induced stress will converge to a measure-valued force term supported only on the interface between $\{\phi=1\}$ and $\{\phi=-1\}$. Moreover, $\tilde{W}(\phi)$ is uniformly bounded in time.

As $\eta \rightarrow 0$, we expect the following equal partition of the energy

$$
\frac{\eta}{2}|\nabla \phi|^{2}=\frac{1}{4 \eta}\left(\phi^{2}-1\right)^{2}
$$

to be held. We point out that this has been rigorously justified in many cases including the Allen-Cahn model (cf. [13,20,21,58,59]).

Let us set

$$
n=\frac{\nabla \phi}{|\nabla \phi|}, \quad a=|\nabla \phi|, \quad H=\nabla \cdot n .
$$


We see that $H$ is the mean curvature of the interface in the limit. With these notations, we can split the induced force term as follows:

$$
\begin{aligned}
\lambda \nabla \cdot(\nabla \phi \otimes \nabla \phi) & =\lambda \nabla \frac{a^{2}}{2}+\lambda \Delta \phi \nabla \phi=\lambda a^{2} H n+\lambda(n \cdot \nabla a) a n+\lambda \nabla \frac{a^{2}}{2} \\
& =\lambda a^{2} H n+\lambda\left(n \cdot \nabla \frac{a^{2}}{2}\right) n+\lambda \nabla \frac{a^{2}}{2}=\lambda a^{2} H n+\lambda\left(n \cdot \nabla \frac{1}{4 \eta^{2}}\left(\phi^{2}-1\right)^{2}\right) n+\lambda \nabla \frac{a^{2}}{2} \\
& =\lambda a^{2} H n+\lambda \frac{1}{\eta^{2}}\left(\phi^{2}-1\right) \phi(n \cdot \nabla \phi) n+\lambda \nabla \frac{a^{2}}{2}=\lambda a^{2} H n+\lambda \frac{1}{\eta^{2}}\left(\phi^{2}-1\right) \phi a n+\lambda \nabla \frac{a^{2}}{2} \\
& =\lambda a^{2} H n+\lambda \frac{1}{\eta^{2}}\left(\phi^{2}-1\right) \phi \nabla \phi+\lambda \nabla \frac{a^{2}}{2}=\lambda a^{2} H n+\lambda \nabla \frac{1}{4 \eta^{2}}\left(\phi^{2}-1\right)^{2}+\lambda \nabla \frac{a^{2}}{2} .
\end{aligned}
$$

Absorbing all the gradient terms in the pressure, we see that the equal partition of the energy gives the pure surface tension on the limiting interface, even though $\phi$ may not be a distance function. This indicates the capillary effect induced by the mixture of two different materials.

Finally, the above calculation also shows that $\lambda / \eta$ is equal to the surface tension constant $m$. Since the mixing width $\eta$ is usually small, so is $\lambda$. However, for each fixed $\eta$ (hence $\lambda$ ), the capillary term stabilizes the system (in fact, it stabilizes the transport of the phase function). Moreover, as $\eta \rightarrow 0$, it is clear that the elastic energy $\int_{\Omega} \tilde{W}(\phi, \nabla \phi) \mathrm{d} x$ converges to the surface energy (area) of the interface.

\subsection{Variable density and viscosity, Boussinesq approximation}

Eqs. (2.1)-(2.3) describe the mixture of two fluids with same density and viscosity. When these material properties are different, we need to modify (2.1)-(2.3) accordingly.

One approach is to define "average" density and viscosity as follows:

$$
\frac{1}{\rho(\phi)}=\frac{1+\phi}{2 \rho_{1}}+\frac{1-\phi}{2 \rho_{2}}, \quad \frac{1}{v(\phi)}=\frac{1+\phi}{2 v_{1}}+\frac{1-\phi}{2 v_{2}},
$$

where $\rho_{1}, \rho_{2}$ are the corresponding density and $\nu_{1}, \nu_{2}$ are the viscosity constants. The reason to choose the harmonic average as in (2.22) is that the solution of the Cahn-Hilliard equation (2.3) does not satisfy the maximal principle. Hence, the linear average cannot be guaranteed to be bounded away from zero. However, due to the $L^{\infty}$-bound of the solution [14], the harmonic averages lead to desired properties. This approach can be replaced using the normal linear averages in the case when (2.3) is replaced by the Allen-Cahn equation for which the solution satisfies the maximal principle.

The modified momentum equation with variable density and viscosity takes the form

$$
(\rho(\phi) u)_{t}+(u \cdot \nabla)(\rho(\phi) u)+\nabla p-\operatorname{div}(v(\phi) D(u))+\lambda \nabla \cdot(\nabla \phi \otimes \nabla \phi)=g(x),
$$

where $g(x)$ is the external body force. As Eq. (2.3) converges to the transport equation (2.5), together with the incompressibility condition (2.2), the density $\rho$ will satisfy the continuity equation:

$$
\rho_{t}+\nabla \cdot(\rho u)=0 .
$$

Another way to model the mixture of different densities is to use the classical Boussinesq approximation, which is the linear version of all different types of average approaches. Here, the "background" density can be treated as a constant $\rho_{0}$ and the difference between the actual density and $\rho_{0}$ will contribute only to the buoyancy force [41]. Hence, the modified momentum equation becomes

$$
\rho_{0}\left(u_{t}+(u \cdot \nabla) u\right)+\nabla p-\operatorname{div}(v D(u))+\lambda \nabla \cdot(\nabla \phi \otimes \nabla \phi)=-(1+\phi) g\left(\rho_{1}-\rho_{0}\right)-(1-\phi) g\left(\rho_{2}-\rho_{0}\right),
$$


where $g$ is the gravitational acceleration. Because of its simplicity in practical implementations, this approach is employed in our numerical Examples 4-6 in Section 5.

\section{Well-posedness and the limiting system}

Following exactly the same arguments as in [42], we can prove the following existence and regularity theorems for the system (2.1)-(2.3). In the following, we assume that all the material parameters $\gamma, \lambda$ and $\eta$ are positive constants.

Theorem 3.1. Assuming that the initial conditions $\left(u_{0}, \phi_{0}\right)$ are such that $u_{0} \in L^{2}(\Omega), \phi_{o} \in H^{1}(\Omega)$ and satisfy the periodic boundary conditions, then, the system (2.1)-(2.3) with the initial condition (2.4) has at least one global weak solution $(u, \phi)$ such that

$$
u \in L^{2}\left(0, T ; H^{1}(\Omega)\right) \cap L^{\infty}\left(0, T ; L^{2}(\Omega)\right), \quad \phi \in L^{2}\left(0, T ; H^{3}(\Omega)\right) \cap L^{\infty}\left(0, T ; H^{1}(\Omega)\right)
$$

for all $0<T<+\infty$.

In addition, we can also derive from higher-order energy estimates the following result:

Theorem 3.2. For any $0<T<+\infty$, there exists $0<T_{1} \leq T$ such that the system (2.1)-(2.3) with the initial conditions (2.4) admits a unique classical solution $(u, d, p)$ in $\left[0, T_{1}\right]$. In particular, $T_{1}=T$ in the two-dimensional case.

As we discussed in the previous sections, the choice of Cahn-Hilliard equation in (2.3), instead of Allen-Cahn equation or other types of regularization for the sharp interface model, is made to maintain the volume fraction $\int_{\Omega} \phi$. In the case where (2.3) is replaced by the Allen-Cahn system:

$$
\phi_{t}+(u \cdot \nabla) \phi-\gamma(\Delta \phi-f(\phi))=0,
$$

we proved in [44] the following result:

Theorem 3.3. For fixed $\gamma$ the system (2.1)-(2.3) and (3.1) will approach, as $\eta \rightarrow 0$, the following auxiliary system:

$$
\begin{aligned}
& u_{t}+(u \cdot \nabla) u+\nabla p-v \operatorname{div} D(u)=g, \\
& \nabla \cdot u=0
\end{aligned}
$$

in the domain away from the interface. The interface evolution satisfies the equation:

$$
z_{t}+(u \cdot \nabla) z=\gamma \Delta z
$$

on $\{x \in \Omega \mid z(x, \cdot)=0\}$, where $z$ is the distance function to the interface (hence $|\nabla z|=1)$. The system also satisfies the traction-free (force balance) boundary condition on the interface $\{x \in \Omega \mid z(x, \cdot)=0\}$ :

$$
[2 v D(u)-p I] \cdot n=m H n,
$$

where $n=\nabla z$ is the normal to the interface, and $H=\Delta z$ is the mean curvature of the interface.

In the above theorem, as $\gamma$ approaches zero, we recover the classical two-phase fluid system. Eq. (3.4) is in fact the motion by mean curvature equation plus a transport (by the velocity $u$ ) term. The convergence is understood in 
the usual viscosity solution sense as in [31]. It is also shown that this system is related to the level set method for tracking the interface [51]. In the proof, we used the transformation

$$
\phi(x, t)=\tanh \left(\frac{z(x, t)}{\eta}\right)
$$

and the fact that as $\eta$ approaches zero, we obtain (formally) Eq. (3.4).

We expect that a corresponding result would hold when the phase equation is of Cahn-Hilliard type. Especially, we believe that in the limit $\eta \rightarrow 0$, the two systems will approach the same limit, that is, the Navier-Stokes equations in each separate domain with the kinematic and traction-free boundary condition on the free interface. We note that such a limit was established in [2] for the Cahn-Hilliard equation without the flow velocity field.

\section{Fourier-spectral approximation}

In this section, we consider Eqs. (2.1)-(2.4) in the domain $\Omega=(0,2 \pi)^{n}(n=2$ or 3$)$ and equipped with periodic conditions in all directions. The choice of periodic boundary condition is legitimate when the boundary effects are negligible (as in the examples in the next section), and is quite appropriate for investigating the correctness and robustness of the present model. Note that the choice of periodic boundary condition leads to a fast and accurate Fourier-spectral method and greatly simplifies the implementation.

Without loss of generality, we assume $\int_{\Omega} \phi_{0} \mathrm{~d} x=0$ and $\int_{\Omega} u_{0} \mathrm{~d} x=0$. For any $r \geq 0$, we set

$$
H_{p}^{r}=\left\{v \in H^{r}(\Omega), v \text { periodic, } \int_{\Omega} v \mathrm{~d} x=0\right\} .
$$

The space $H_{p}^{r}$ is equipped with the semi-norm $|\cdot|_{r}=|\cdot|_{H^{r}(\Omega)}$ and the norm $\|\cdot\|_{r}=\|\cdot\|_{H^{r}(\Omega)}$. We set in particular $\|\cdot\|=\|\cdot\|_{0}$. It is well-known that

$$
\|\phi\|_{\alpha} \leq c\|\phi\|_{\beta} \leq c|\phi|_{\beta}, \quad \forall v \in H_{p}^{\beta}(\alpha<\beta) .
$$

We also denote

$$
H=\left\{v \in\left(H_{p}^{0}\right)^{n}: \nabla \cdot v=0\right\}, \quad V=\left\{v \in\left(H_{p}^{1}\right)^{n}: \nabla \cdot v=0\right\} .
$$

For any $v, w \in H_{p}^{1}$, let

$$
(D(v), D(w))=\int_{\Omega} D(v) \cdot D(w) \mathrm{d} x .
$$

We have

$$
(D(v), D(v)) \geq \frac{1}{2}|v|_{1}^{2} .
$$

A direct calculation shows that for any $v \in H_{p}^{2}$,

$$
\nabla \cdot(\nabla v \otimes \nabla v)=\nabla\left(\frac{1}{2}|\nabla v|^{2}\right)+(\nabla v)^{\mathrm{T}} \Delta v .
$$

Therefore,

$$
(\nabla \cdot(\nabla v \otimes \nabla v), w)=\left(\Delta v^{\mathrm{T}}(\nabla v), w\right), \quad v \in H_{p}^{2}, \quad w \in H .
$$

One can also easily check the following skew-symmetric property:

$$
((v \cdot \nabla) z, w)=-((v \cdot \nabla) w, z), \quad \forall v \in V, \quad w, z \in H_{p}^{1},
$$


and similarly,

$$
((v \cdot \nabla) w, f(w))=(v, \nabla F(\phi))=0, \quad \forall v \in V, \quad w \in H_{p}^{1} .
$$

To introduce the Fourier-spectral approximation, we set

$$
\begin{aligned}
& P_{M}=\operatorname{span}\{\sin m x, \cos m x, m=1,2, \ldots, M\}, \\
& \mathcal{P}_{M}=P_{M} \times P_{M}(n=2), \quad \mathcal{P}_{M}=P_{M} \times P_{M} \times P_{M}(n=3), \quad V_{M}=\mathcal{P}_{M}^{n} \cap V .
\end{aligned}
$$

Let $\pi_{M}: H \rightarrow \mathcal{P}_{M}$ be the $H_{p}^{0}$-orthogonal projector. The Fourier-spectral method for (2.1)-(2.4) can be formulated as follows:

Find $\left(u_{M}, \phi_{M}\right) \in V_{M} \times \mathcal{P}_{M}$ such that

$$
\begin{aligned}
& \frac{\mathrm{d}}{\mathrm{d} t}\left(u_{M}, v\right)+\left(\left(u_{M} \cdot \nabla\right) u_{M}, v\right)+v\left(D\left(u_{M}\right), D(v)\right)+\lambda\left(\nabla \cdot\left(\nabla \phi_{M} \otimes \nabla \phi_{M}\right), v\right)=0, \quad \forall v \in V_{M}, \\
& \frac{\mathrm{d}}{\mathrm{d} t}\left(\phi_{M}, \psi\right)+\left(\left(u_{M} \cdot \nabla\right) \phi_{M}, \psi\right)-\gamma\left(\nabla\left(\Delta \phi_{M}-f\left(\phi_{M}\right)\right), \nabla \psi\right)=0, \quad \forall \psi \in \mathcal{P}_{M},
\end{aligned}
$$

with $u_{M}(x, 0)=\pi_{M} u_{0}(x)$ and $\phi_{M}(x, 0)=\pi_{M} \phi_{0}(x)$. We note that in practice, different values of $M$ may be used for $V_{M}$ and $\mathcal{P}_{M}$.

A complete stability and error analysis for (4.10) is beyond the scope of this paper whose main purpose is to propose and justify a phase field model for the mixture of two incompressible fluids. Nevertheless, we prove below a priori estimates which are critical for establishing the well-posedness and error estimates for (4.10).

Theorem 4.1. Let $\left(u_{M}, \phi_{M}\right)$ be any solution of the system (4.10). For any given $\delta<4$, there exist two positive constants $c_{1}, c_{2}(\delta)$ independent of any function, $M$ and $\eta$ such that:

(i) three-dimensional case: for all $t \in\left[0, T_{1}\right)$, we have

$$
\begin{aligned}
& \left\|u_{M}(t)\right\|^{2}+\lambda\left|\phi_{M}(t)\right|_{1}^{2} \leq \frac{\left\|u_{M}(0)\right\|^{2}+\lambda\left|\phi_{M}(0)\right|_{1}^{2}}{\left(1-\left(t / T_{1}\right)\right)^{1 / 4}}, \\
& \int_{0}^{t}\left(\nu\left|u_{M}(s)\right|_{1}^{2}+\lambda \gamma\left\|\nabla \Delta \phi_{M}(s)\right\|^{2}\right) \mathrm{d} s \leq\left(\left\|u_{M}(0)\right\|^{2}+\lambda\left\|\phi_{M}(0)\right\|_{1}^{2}\right)+\frac{c_{1} \eta^{-8} t\left(\left\|u_{M}(0)\right\|^{2}+\lambda\left|\phi_{M}(0)\right|_{1}^{2}\right)^{5}}{\left(1-\left(t / T_{1}\right)\right)^{5 / 4}},
\end{aligned}
$$

where

$$
T_{1}=\frac{\eta^{8}}{4 c_{1}\left(\left\|u_{M}(0)\right\|^{2}+\lambda\left|\phi_{M}(0)\right|_{1}^{2}\right)^{4}} .
$$

(ii) two-dimensional case: for all $t \in\left[0, T_{2}\right)$, we have

$$
\begin{aligned}
& \left\|u_{M}(t)\right\|^{2}+\lambda\left|\phi_{M}(t)\right|_{1}^{2}+2 \lambda \int_{\Omega} F\left(\phi_{M}(t)\right) \mathrm{d} x \leq \frac{\left\|u_{M}(0)\right\|^{2}+\lambda\left|\phi_{M}(0)\right|_{1}^{2}+2 \lambda \int_{\Omega} F\left(\phi_{M}(0)\right) \mathrm{d} x}{\left(1-\left(t / T_{2}\right)\right)^{1 / 3}}, \\
& \int_{0}^{t}\left(v\left|u_{M}(s)\right|_{1}^{2}+\lambda \gamma\left\|\nabla\left(\Delta \phi_{M}(s)-\pi_{M} f\left(\phi_{M}(s)\right)\right)\right\|^{2}\right) \mathrm{d} s \\
& \leq\left(\left\|u_{M}(0)\right\|^{2}+\lambda\left\|\phi_{M}(0)\right\|_{1}^{2}+2 \lambda \int_{\Omega} F\left(\phi_{M}(0)\right) \mathrm{d} x\right) \\
& \quad+\frac{c_{2} \eta^{-4} M^{\delta-2} t\left(\left\|u_{M}(0)\right\|^{2}+\lambda\left|\phi_{M}(0)\right|_{1}^{2}+2 \lambda \int_{\Omega} F\left(\phi_{M}(0)\right) \mathrm{d} x\right)^{4}}{\left(1-\left(t / T_{2}\right)\right)^{4 / 3}}
\end{aligned}
$$


where

$$
T_{2}=\frac{\eta^{4}}{c_{2}(\delta) M^{2-\delta}\left(\left\|u_{M}(0)\right\|^{2}+\lambda\left|\phi_{M}(0)\right|_{1}^{2}+2 \lambda \int_{\Omega} F\left(\phi_{M}(0)\right) \mathrm{d} x\right)^{3}} .
$$

Proof. We take $v=2 u_{M}$ and $\psi=-2 \lambda \Delta \phi_{M}$ in (4.10). Using (4.4), (4.6) and (4.7), we obtain that

$$
\frac{\mathrm{d}}{\mathrm{d} t}\left\|u_{M}\right\|^{2}+v\left|u_{M}\right|_{1}^{2}+2 \lambda\left(\left(\Delta \phi_{M}^{\mathrm{T}}\right) \nabla \phi_{M}, u_{M}\right)=0
$$

and

$$
\lambda \frac{\mathrm{d}}{\mathrm{d} t}\left|\phi_{M}\right|_{1}^{2}-2 \lambda\left(\left(u_{M} \cdot \nabla\right) \phi_{M}, \Delta \phi_{M}\right)+2 \lambda \gamma\left\|\nabla \Delta \phi_{M}\right\|^{2}=2 \lambda \gamma\left(\nabla f\left(\phi_{M}\right), \nabla \Delta \phi_{M}\right) .
$$

Below, we shall use $c$ to denote a generic positive constant independent of any function, $\eta$ and $M$.

Using the Sobolev inequality (cf. [32,38])

$$
\|v\|_{L^{\infty}} \leq c\|v\|_{1}^{3 / 4}\|v\|_{3}^{1 / 4}, \quad \forall v \in H_{p}^{3} \quad(n \leq 3),
$$

the Hölder's inequality and (4.2), we find

$$
\begin{aligned}
2 \lambda \gamma\left(\nabla f\left(\phi_{M}\right), \nabla \Delta \phi_{M}\right) & =2 \lambda \gamma\left(f^{\prime}\left(\phi_{M}\right) \nabla \phi_{M}, \nabla \Delta \phi_{M}\right) \leq c\left\|f^{\prime}\left(\phi_{M}\right)\right\|_{L^{\infty}}\left\|\phi_{M}\right\|_{3}\left\|\phi_{M}\right\|_{1} \\
& \leq \frac{c}{\eta^{2}}\left(\|\phi\|_{L^{\infty}}^{2}+1\right)\left\|\phi_{M}\right\|_{3}\left\|\phi_{M}\right\|_{1} \leq \frac{c}{\eta^{2}}\left(\left\|\phi_{M}\right\|_{1}^{3 / 2}\left\|\phi_{M}\right\|_{3}^{1 / 2}+1\right)\left\|\phi_{M}\right\|_{3}\left\|\phi_{M}\right\|_{1} \\
& \leq \lambda \gamma\left\|\nabla \Delta \phi_{M}\right\|^{2}+\frac{c}{\eta^{8}}\left|\phi_{M}\right|_{1}^{10}+\frac{c}{\eta^{4}}\left|\phi_{M}\right|_{1}^{2} .
\end{aligned}
$$

Combining (4.11), (4.12) and (4.14), we obtain

$$
\begin{aligned}
& \frac{\mathrm{d}}{\mathrm{d} t}\left(\left\|u_{M}(t)\right\|^{2}+\lambda\left|\phi_{M}(t)\right|_{1}^{2}\right)+v\left|u_{M}(t)\right|_{1}^{2}+\lambda \gamma\left\|\nabla \Delta \phi_{M}(t)\right\|^{2} \leq \frac{c}{\eta^{4}}\left|\phi_{M}(t)\right|_{1}^{2}+\frac{c}{\eta^{8}}\left|\phi_{M}(t)\right|_{1}^{10} \\
& \quad \leq \frac{c_{1}}{\eta^{8}}\left(1+\left\|u_{M}(t)\right\|^{2}+\lambda\left|\phi_{M}(t)\right|_{1}^{2}\right)^{5}
\end{aligned}
$$

We derive the first result by applying Lemma 4.1 with $m=5, \beta=c_{1} / \eta^{8}$ and

$$
y(t)=1+\left\|u_{M}(t)\right\|^{2}+\lambda\left|\phi_{M}(t)\right|_{1}^{2}, \quad b(t)=v\left|u_{M}(t)\right|_{1}^{2}+\lambda \gamma\left\|\nabla \Delta \phi_{M}(t)\right\|^{2} .
$$

The above result is valid for both the three-dimensional and two-dimensional cases. However, for the two-dimensional case, an improved result can be obtained as follows.

Taking $\psi=-2 \lambda\left(\Delta \phi_{M}-\pi_{M} f\left(\phi_{M}\right)\right)$ in (4.10), instead of (4.12), we obtain

$$
\begin{aligned}
& \frac{\mathrm{d}}{\mathrm{d} t}\left(\lambda\left|\phi_{M}\right|_{1}^{2}+2 \lambda \int_{\Omega} F\left(\phi_{M}\right) \mathrm{d} x\right)-2 \lambda\left(\left(u_{M} \cdot \nabla\right) \phi_{M}, \Delta \phi_{M}\right)+2 \lambda\left(\left(u_{M} \cdot \nabla\right) \phi_{M}, \pi_{M} f\left(\phi_{M}\right)\right) \\
& \quad+2 \lambda \gamma\left\|\nabla\left(\Delta \phi_{M}-\pi_{M} f\left(\phi_{M}\right)\right)\right\|^{2}=0 .
\end{aligned}
$$

Let $I$ be the identity operator, and $g(z)=\left(1 / \eta^{2}\right)|z|^{2} z$. Thus, the sum of (4.11)-(4.16) leads to

$$
\begin{aligned}
& \frac{\mathrm{d}}{\mathrm{d} t}\left(\left\|u_{M}\right\|^{2}+\lambda\left|\phi_{M}\right|_{1}^{2}+2 \lambda \int_{\Omega} F\left(\phi_{M}\right) \mathrm{d} x\right)+2 v\left|u_{M}\right|_{1}^{2}+2 \lambda \gamma\left\|\nabla\left(\Delta \phi_{M}-\pi_{M} f\left(\phi_{M}\right)\right)\right\|^{2} \\
& \quad \leq 2 \lambda\left(\left(u_{M} \cdot \nabla\right) \phi_{M},\left(I-\pi_{M}\right) g\left(\phi_{M}\right)\right) .
\end{aligned}
$$


We recall that for any $v \in H_{p}^{r}$ and $0 \leq \mu \leq r$, we have

$$
\left\|\pi_{M} v-v\right\|_{\mu} \leq c M^{\mu-r}\|v\|_{r}
$$

Therefore,

$$
\left\|\left(I-\pi_{M}\right) g\left(\phi_{M}\right)\right\| \leq c M^{-1}\left\|g\left(\phi_{M}\right)\right\|_{1} .
$$

For any given $\delta<4$, we set $p=4 / \delta$. Using the Hölder's inequality and the Sobolev embedding theorem (in the two-dimensional case), we find

$$
\begin{aligned}
\left\|g\left(\phi_{M}\right)\right\|_{1}^{2} & \leq c\left|g\left(\phi_{M}\right)\right|_{1}^{2} \leq \frac{c}{\eta^{4}}\left|\phi_{M}^{2} \nabla \phi_{M}\right|_{1}^{2} \leq \frac{c}{\eta^{4}}\left\|\phi_{M}^{4}\right\|_{L^{p}}\left\|\left(\nabla \phi_{M}\right)^{2}\right\|_{L^{p /(p-1)}} \\
& =\frac{c}{\eta^{4}}\left\|\phi_{M}\right\|_{L^{4 p}}^{4}\left\|\nabla \phi_{M}\right\|_{L^{2 p /(p-1)}}^{2} \leq \frac{c}{\eta^{4}}\left\|\phi_{M}\right\|_{1}^{4}\left\|\nabla \phi_{M}\right\|_{1 / p}^{2} \leq \frac{c M^{2 / p}}{\eta^{4}}\left\|\phi_{M}\right\|_{1}^{6},
\end{aligned}
$$

where in the last step we have used the inverse inequality

$$
\left\|v_{M}\right\|_{r} \leq c M^{r}\left\|v_{M}\right\|, \quad \forall v_{M} \in \mathcal{P}_{M} .
$$

Now, we combine (4.19) and (4.20) and use the Hölder's inequality and the Sobolev embedding theorem again to get

$$
\begin{aligned}
& 2 \lambda\left|\left(\left(u_{M} \cdot \nabla\right) \phi_{M},\left(I-\pi_{M}\right) g\left(\phi_{M}\right)\right)\right| \\
& \quad \leq 2 \lambda\left\|u_{M}\right\|_{L^{2 p}}\left\|\nabla \phi_{M}\right\|_{L^{2 p /(p-1)}}\left\|\left(I-\pi_{M}\right) g\left(\phi_{M}\right)\right\| \\
& \quad \leq c M^{(2 / p)-1} \eta^{-2}\left\|u_{M}\right\|_{1}\left\|\phi_{M}\right\|_{1}^{4} \leq v\left|u_{M}\right|_{1}^{2}+c M^{(4 / p)-2} \eta^{-4}\left\|\phi_{M}\right\|_{1}^{8} .
\end{aligned}
$$

Substituting (4.22) into (4.17), we find

$$
\begin{aligned}
& \frac{\mathrm{d}}{\mathrm{d} t}\left(\left\|u_{M}\right\|^{2}+\lambda\left|\phi_{M}\right|_{1}^{2}+2 \lambda \int_{\Omega} F\left(\phi_{M}\right) \mathrm{d} x\right)+v\left|u_{M}\right|_{1}^{2}+2 \lambda \gamma\left\|\nabla\left(\Delta \phi_{M}-\pi_{M} f\left(\phi_{M}\right)\right)\right\|^{2} \\
& \quad \leq c_{2}(\delta) \eta^{-4} M^{(4 / p)-2}\left\|\phi_{M}\right\|_{1}^{8} .
\end{aligned}
$$

We can then conclude by applying Lemma 4.1 below to the above with $m=4, \beta=c_{2} \eta^{-4} M^{(4 / p)-2}$ and

$$
y(t)=\left\|u_{M}\right\|^{2}+\lambda\left|\phi_{M}\right|_{1}^{2}+2 \lambda \int_{\Omega} F\left(\phi_{M}\right) \mathrm{d} x, \quad b(t)=v\left|u_{M}\right|_{1}^{2}+2 \lambda \gamma\left\|\nabla\left(\Delta \phi_{M}-\pi_{M} f\left(\phi_{M}\right)\right)\right\|^{2}
$$

Lemma 4.1. Let $\beta>0, m>1$, and $y(t), b(t)$ be two non-negative functions satisfying

$$
y^{\prime}(t)+b(t) \leq \beta y^{m}(t), \quad t \in(0, T) .
$$

Then, for $t \in\left[0, T_{0}\right)$ with $T_{0}=\min \left(1 /\left((m-1) \beta y^{m-1}(0)\right), T\right)$, we have

$$
y(t) \leq \frac{y(0)}{\left(1-(m-1) \beta y^{m-1}(0) t\right)^{1 /(m-1)}}, \quad \int_{0}^{t} R(s) \mathrm{d} s \leq y(0)+\beta t \frac{y(0)^{m}}{\left(1-(m-1) \beta y^{m-1}(0) t\right)^{m /(m-1)}} .
$$

Proof. We sketch the proof below for the readers' convenience.

Let $v(t)=y^{-m-1}(t)$. We derive from (4.25) that

$$
v^{\prime}(t) \geq-(m-1) \beta \quad \text { and } \quad v(t) \geq v(0)-(m-1) \beta t .
$$

The first result follows directly from the above. Integrating (4.25) and taking into account the first result, we obtain the second result. 
Remark 4.1. With the a priori estimates established in the above theorem, one can follow essentially the same standard procedure as in [27] to prove the well-posedness of the system (4.10) and to establish an error estimate of spectral-type, namely, the convergence rate of the Fourier-approximation is only limited by the smoothness of the solution.

We would like to point out that the above analysis can be essentially extended to other admissible boundary conditions.

\section{Numerical results}

We implemented a second-order semi-implicit time discretization scheme for the Fourier-spectral system (4.10) in the two-dimensional case. Thanks to the periodic boundary conditions, the pressure can be easily eliminated from the system, and the Laplace and biharmonic operators are reduced to, in Fourier space, diagonal operators. Thus, at each time step, the linear systems (for the discrete Fourier coefficients of the unknown functions) to be solved are diagonal systems and the computational cost is dominated by the evaluation of the nonlinear terms in (4.10) which, in actual computations, are computed using the so-called pseudospectral/collocation approach with fast Fourier transform (FFT).

Below, we present several numerical experiments using this code. In all computations, we have fixed the physical parameters to be

$$
\eta=0.02, \quad \lambda=0.1, \quad v=0.1, \quad \gamma=0.1
$$

and the computational parameters to be $M=128$ and $\mathrm{d} t=0.005$. The initial condition for $u$ is taken to be zero in all computations while the initial condition for $\phi$ is specified in each example. We recall that $\eta$ is the capillary width (mixing region) of the fluids, $\lambda / \eta$ is the surface tension constant, $v$ is the viscosity and $\gamma$ is the "elastic" relaxation time.

Example 1 (Surface tension effects). This test exhibits the surface tension effects of the model. We start with a rectangular bubble, i.e., $\phi=1$ inside the bubble and $\phi=-1$ outside the bubble. The rectangular bubble quickly deforms into a circular bubble due to the surface tension. In fact, if we choose $\lambda=0$ (i.e., no fluid in the system), the bubble will not deform. Also, we notice that the volume of the bubble is preserved (Fig. 5.1).

Example 2 (Surface tension effects and Allen-Cahn dissipation). In this test, we choose the same initial condition as in Example 1 but we replace the Cahn-Hilliard system (2.3) by the Allen-Cahn system. We notice that the rectangular bubble still deforms into a circular bubble while the size of the bubble shrinks (eventually it shrinks to zero) due to the dissipative mechanism in the Allen-Cahn system.

Notice that in both Examples 1 and 2, the shape of the bubble vibrates tangentially before it becomes a circular bubble (the preferred configuration). This tangential vibration is attributed to the so-called T-modes of the spheric normal modes (cf. [61]). This illustrates that our model captures another important special effect of the surface tension (Fig. 5.2).

Example 3 (Coalescence of two kissing bubbles). We start with two kissing bubbles. As time evolves, the two bubbles coalesces into one big bubble. This is the combination of the surface tension effect and the elastic effect from the phase equation. We note that if we start with two non-kissing bubbles of the same size, the two bubbles 

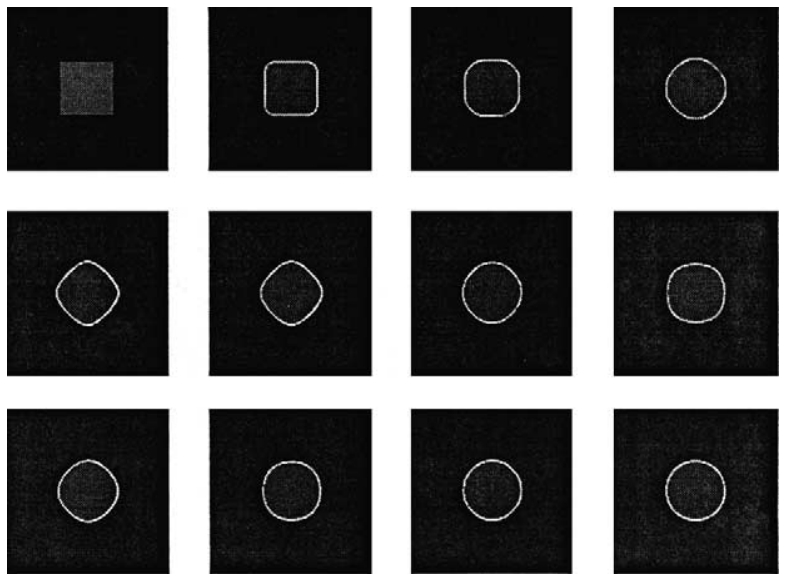

Fig. 5.1. Example 1: phase evolution at $t=0,0.1,0.2,0.3,0.4,0.5,0.6,0.7,1.2,1.4,1.7,2.5$.
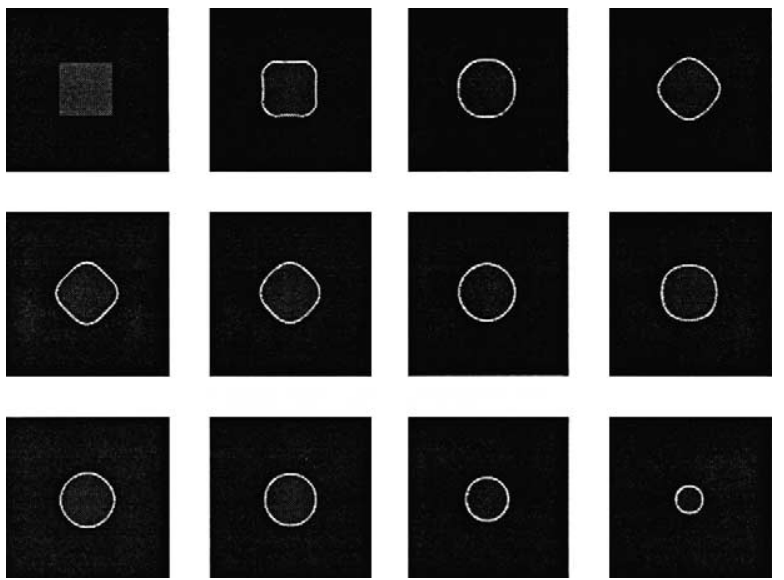

Fig. 5.2. Example 2: (Allen-Cahn) phase evolution at $t=0,0.1,0.2,0.3,0.4,0.5,0.6,0.7,1.2,1.6,3.0,5.0$.
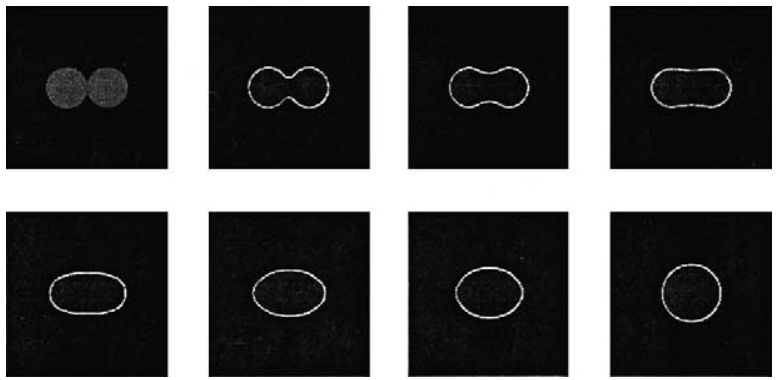

Fig. 5.3. Example 3: phase evolution at $t=0,0.1,0.2,0.3,0.4,0.5,0.6,0.8$. 

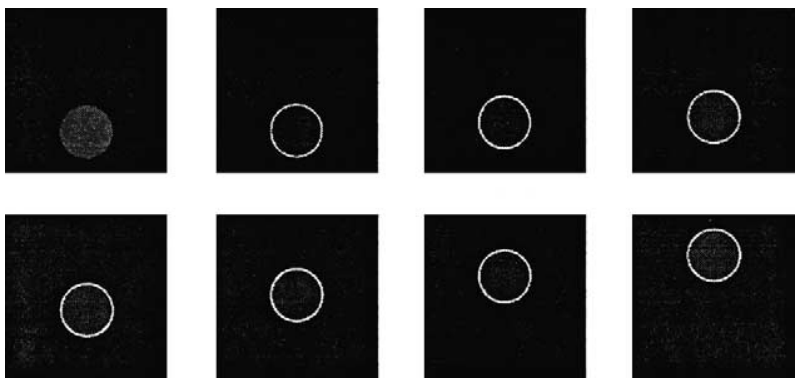

Fig. 5.4. Example 4: phase evolution at $t=0,4.0,5.0,6.0,7.5,9.0,10.5,12.0$.
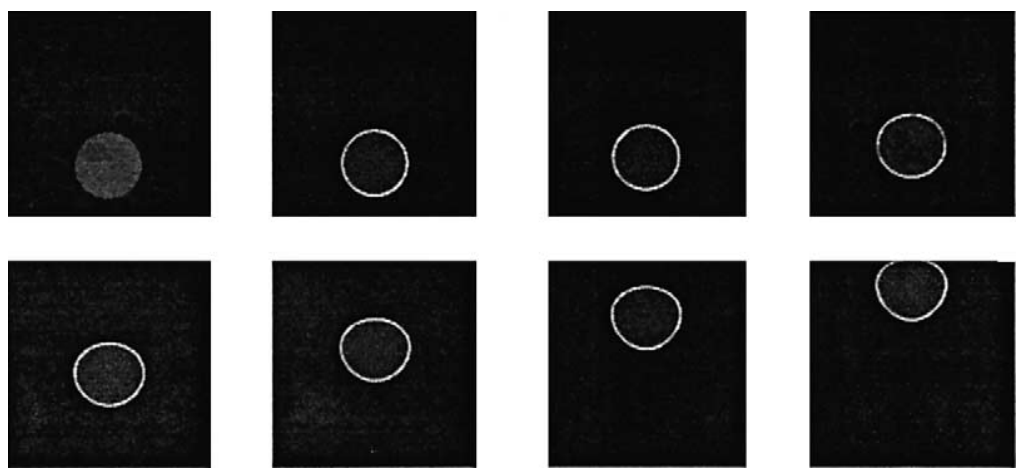

Fig. 5.5. Example 5: phase evolution at $t=0,1.2,1.8,2.5,3.3,4.0,4.8,5.5$.

will not move since the dynamics of Cahn-Hilliard is static (i.e., the chemical potential and the curvature are the same).

In the next three examples, we use the Boussinesq approximation (2.25) to model the case where the two fluids have different densities. We rewrite the right-hand side of (2.25) as

$$
-g\left(2 \rho_{0}+\rho_{1}+\rho_{2}\right)-g \phi\left(\rho_{1}-\rho_{2}\right) .
$$

The first term is a constant vector which can be absorbed into the pressure so we only have to consider the second term. In all three examples below, we set $\rho_{1}-\rho_{2}=-1$ (Fig. 5.3).
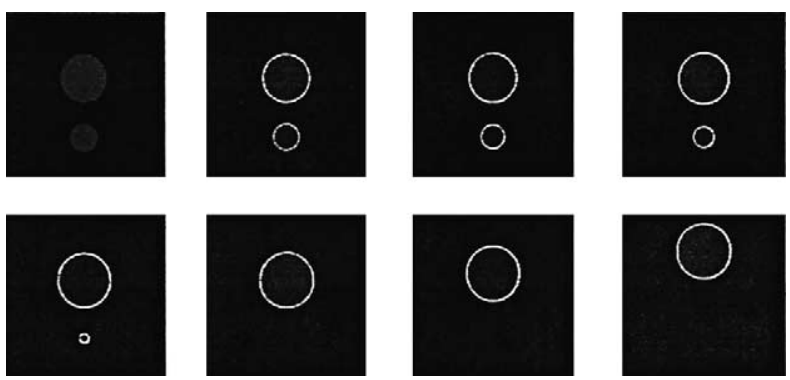

Fig. 5.6. Example 6: phase evolution at $t=0,0.1,0.2,0.3,0.5,1.0,6.0,9.0$. 
Example 4 (Boussinesq approximation, Case A). We start with a circular bubble near the bottom of the domain. The density of the bubble is lighter than the density of the surrounding fluid. The gravitational constant vector is taken to be $g=(0.1,0)^{t}$. The bubble rises as expected due to the gravity differential (Fig. 5.4).

Example 5 (Boussinesq approximation, Case B). The situation is the same as in Example 4 except that the gravitational constant vector is taken to be $g=(1,0)^{t}$. Thus, the gravity differential is 10 times larger than the previous case.

We notice that the bubble deforms as it rises. The deformation of the shape of the bubble indicates the influence of the flow field. The shape deformation is not visually noticeable in the previous case due to the much smaller gravity differential (Fig. 5.5).

Example 6 (Boussinesq approximation, Case C). We start with two circular bubbles of different sizes at different heights. The density of the bubbles is lighter than the density of the surrounding fluid. Here, the gravitational constant vector is taken to be $g=(0.1,0)^{t}$.

Notice that the small bubble is absorbed by the big bubble before any noticeable rise. This phenomenon, i.e., the big bubble absorbs the small bubble, is purely due to the Cahn-Hilliard equation, since the curvature of the bubbles serves as the chemical potential in the dynamics of the phase function. The rate of this process is determined by the elastic relaxation time $\gamma$ (Fig. 5.6).

\section{Concluding remarks}

We presented in this paper a phase field model for the mixture of two incompressible fluids. The model consists of a momentum equation with an extra "elastic" term due to the mixing of different materials and a Cahn-Hilliard equation with the corresponding transport term. We illustrated that such a system converges to the usual two phase fluid system as the mixing region shrinks to an interface. We note that the derivation of the model provides a general framework to incorporate the elastic effects in different complex fluids.

We analyzed a semi-discrete Fourier-spectral method for the numerical approximation of this system and implemented a semi-implicit scheme for the time discretization. We presented several illustrative numerical examples which exhibited various physical mechanisms of the model and demonstrated its robustness and versatility. These examples demonstrate that the proposed model captures many interesting surface tension effects that are of great interest in the theory of mixtures and interfaces.

In upcoming works, we will investigate the cases with other admissible boundary conditions, with variable viscosities, and eventually the more challenging cases such as solid-liquid and liquid-air mixtures. We also plan to study different interfacial dynamics (configurations) for the mixtures of more complicated materials, such as liquid crystals, polymeric materials and viscoelastic solids.

\section{Acknowledgements}

The work of C. Liu is supported in part by National Science Foundation Grant DMS-9972040 and that of J. Shen is supported in part by National Science Foundation Grant DMS-0074283.

\section{References}

[1] R. Abraham, J.E. Marsden, Foundations of Mechanics, 2nd ed., revised and enlarged, with the assistance of T. Ratiu and R. Cushman, Benjamin/Cummings, Advanced Book Program, Reading, MA, 1978. 
[2] N.D. Alikakos, P.W. Bates, X.F. Chen, Convergence of the Cahn-Hilliard equation to the Hele-Shaw model, Arch. Rational Mech. Anal. 128 (1994) 165-205.

[3] D.M. Anderson, G.B. McFadden, A diffuse-interface description of internal waves in a near-critical fluid, Phys. Fluids 9 (1997).

[4] D.M. Anderson, G.B. McFadden, A.A. Wheeler, Diffuse-interface methods in fluid mechanics, in: Annual Review of Fluid Mechanics, vol. 30, Annual Reviews, Palo Alto, CA, 1998, pp. 139-165.

[5] V.I. Arnold, Mathematical Methods of Classical Mechanics, Springer, New York, 1989 [K. Vogtmann, A. Weinstein, translators, from the 1974 Russian original, corrected reprint of the second (1989) edition].

[6] G.K. Batchelor, An Introduction to Fluid Dynamics, Cambridge University Press, Cambridge, 1999.

[7] A.N. Beris, B.J. Edwards, Thermodynamics of Flow Systems, with Internal Microstructure, Oxford Science Publication, 1994.

[8] R.B. Bird, R.C. Armstrong, O. Hassager, Dynamics of Polymeric Liquids, vol. 1, Fluid Mechanics, Wiley/Interscience, New York, 1987.

[9] T. Blesgen, A generalization of the Navier-Stokes equations to two phase flow, Preprint, 2000.

[10] F. Boyer, Mathematical study of multi-phase flow under shear through order parameter formulation, Asymptot. Anal. 20 (1999) 175-212.

[11] F. Boyer, A theoretical and numerical model for the study of incompressible mixture flows, Comput. Fluids 31 (2002) 41-68.

[12] L. Bronsard, R. Kohn, Motion by mean curvature as the singular limit of Ginzburgh-Landau model, J. Diff. Eqns. 90 (1991) $211-237$.

[13] L. Bronsard, R. Kohn, On the slowness of phase boundary motion in one space dimension, Comm. Pure Appl. Math. 43 (1990) $983-997$.

[14] L.A. Caffarelli, N.E. Muler, An $L^{\infty}$ bound for solutions of the Cahn-Hilliard equation, Arch. Rational Mech. Anal. 133 (1995) 129-144.

[15] G. Caginalp, X.F. Chen, Phase field equations in the singular limit of sharp interface problems, in: On the Evolution of Phase Boundaries (Minneapolis, MN, 1990-1991), Springer, New York, 1992, pp. 1-27.

[16] J.W. Cahn, S.M. Allen, A microscopic theory for domain wall motion and its experimental verification in $\mathrm{Fe}-\mathrm{Al}$ alloy domain growth kinetics, J. Phys. Colloque C7 (1978) C7-C51.

[17] J.W. Cahn, J.E. Hillard, Free energy of a nonuniform system. I. Interfacial free energy, J. Chem. Phys. 28 (1958) $258-267$.

[18] P.M. Chaikin, T.C. Lubensky, Principles of Condensed Matter Physics, Cambridge, 1995.

[19] Y.C. Chang, T.Y. Hou, B. Merriman, S. Osher, A level set formulation of Eulerian interface capturing methods for incompressible fluid flows, J. Comput. Phys. 124 (1996) 449-464.

[20] X.F. Chen, Spectrum for the Allen-Cahn, Cahn-Hilliard, and phase-field equations for generic interfaces, Comm. Partial Diff. Eqns. 19 (1994) 1371-1395.

[21] X.F. Chen, Generation and propagation of interfaces in reaction-diffusion systems, Trans. Am. Math. Soc. 334 (1992).

[22] K.A. Cliffe, S.J. Tavener, Marangoni-Bénard convection with a deformable free surface, J. Comput. Phys. 145 (1998) $193-227$.

[23] P.G. de Gennes, J. Prost, The Physics of Liquid Crystals, Oxford University Press, Oxford, 1993.

[24] I.V. Denisova, V.A. Solonnikov, Solvability of a linearized problem on the motion of a drop in a fluid flow, Zap. Nauchn. Sem. Leningrad. Otdel. Mat. Inst. Steklov. (LOMI), 171 (1989).

[25] D.L. Denny, R.L. Pego, Models of low-speed flow for near-critical fluids with gravitational and capillary effects, Quart. Appl. Math. 58 (2000) 103-125.

[26] M. Doi, S.F. Edwards, The Theory of Polymer Dynamics, Oxford Science Publication, 1986.

[27] Q. Du, J. Shen, B.Y. Guo, Fourier spectral approximation to a dissipative system modeling the flow of liquid crystals, SIAM J. Numer. Anal. 39 (2001) 735-762.

[28] J.E. Dunn, J. Serrin, On the thermomechanics of interstitial working, Arch. Rational Mech. Anal. 88 (1985) 95-133.

[29] W. E, P. Palffy-Muhoray, Phase separation in incompressible systems, Phys. Rev. E 55 (3) (1997) R3844-R3846.

[30] D.A. Edwards, H. Brenner, D.T. Wasan, Interfacial Transport Process and Rheology, Butterworths/Heinemann, London, 1991.

[31] L.C. Evans, H.M. Soner, P.E. Souganidis, Phase transitions and generalized motion by mean curvature, Comm. Pure Appl. Math. 45 (1992) 1097-1123.

[32] D. Gilbarg, N.S. Trudinger, Elliptic Partial Differential Equations of Second Order, Springer, Berlin, 1983.

[33] M.E. Gurtin, D. Polignone, J. Viñals, Two-phase binary fluids and immiscible fluids described by an order parameter, Math. Models Meth. Appl. Sci. 6 (1996) 815-831.

[34] R.S. Hamilton, The inverse function theorem of Nash and Moser, Bull. Am. Math. Soc. (NS) 7 (1982) 65-222.

[35] R.S. Hamilton, Three-manifolds with positive Ricci curvature, J. Diff. Geom. 17 (1982) 255-306.

[36] D. Jacqmin, Calculation of two-phase Navier-Stokes flows using phase-field modeling, J. Comput. Phys. 155 (1999) 96-127.

[37] V.V. Krotov, A.I. Rusanov, Physicochemical Hydrodynamics of Capillary Systems, Imperial College Press, London, 1999.

[38] O.A. Ladyzhenskaya, The Mathematical Theory of Viscous Incompressible Fluid, Gordon and Breach, London, 1969.

[39] R.G. Larson, The Structure and Rheology of Complex Fluids, Oxford, 1995.

[40] V. Levich, Physicochemical Hydrodynamics, Prentice-Hall, Englewood Cliffs, NJ, 1962.

[41] J. Lighthill, Waves in Fluids, Cambridge, 1978.

[42] F.H. Lin, C. Liu, Nonparabolic dissipative systems, modeling the flow of liquid crystals, Comm. Pure Appl. Math. XLVIII (1995) $501-537$.

[43] F.H. Lin, C. Liu, Static and dynamic theories of liquid crystals, J. Partial Diff. Eqns. 14 (2001) 289-330.

[44] C. Liu, S. Shkoller, Variational phase field model for the mixture of two fluids, Preprint, 2001.

[45] C. Liu, S.J. Tavener, N.J. Walkington, A variational phase field model for Marangoni-Bénard convection with a deformable free surface, Preprint, 2001.

[46] C. Liu, N.J. Walkington, An Eulerian description of fluids containing visco-hyperelastic particles, Arch. Rat. Mech. Anal. 159 (2001) 229-252. 
[47] J. Lowengrub, L. Truskinovsky, Quasi-incompressible Cahn-Hilliard fluids and topological transitions, R. Soc. Lond. Proc. Ser. A Math. Phys. Eng. Sci. 454 (1998) 2617-2654.

[48] G.B. McFadden, A.A. Wheeler, D.M. Anderson, Thin interface asymptotics for an energy/entropy approach to phase-field models with unequal conductivities, Physica D 144 (2000).

[49] G.B. McFadden, A.A. Wheeler, R.J. Braun, S.R. Coriell, R.F. Sekerka, Phase-field models for anisotropic interfaces, Phys. Rev. E 48 (3) (1993) 2016-2024.

[50] W.W. Mullins, R.F. Sekerka, On the thermodynamics of crystalline solids, J. Chem. Phys. 82 (1985).

[51] S. Osher, J. Sethian, Fronts propagating with curvature dependent speed: algorithms based on Hamilton Jacobi formulations, J. Comput. Phys. 79 (1988) 12-49.

[52] R.F. Probstein, Physicochemical Hydrodynamics: An Introduction, Wiley, New York, 1994.

[53] T. Qian, X.P. Wang, P. Sheng, Molecular scale contact line hydrodynamics of immiscible flows, Preprint, 2002.

[54] A.D. Rey, Viscoelastic theory for nematic interfaces, Phys. Rev. E 61 (2000) 1540-1549.

[55] J. Rubinstein, P. Sternberg, J.B. Keller, Fast reaction, slow diffusion, and curve shortening, SIAM J. Appl. Math. 49 (1989) $116-133$.

[56] R. Schoen, K. Uhlenbeck, A regularity theory for harmonic maps, J. Diff. Geom. 17 (1982) 307-335.

[57] R. Schoen, K. Uhlenbeck, Regularity of minimizing harmonic maps into the sphere, Invent. Math. 78 (1984) 89-100.

[58] H.M. Soner, Ginzburg-Landau equation and motion by mean curvature. I. Convergence, J. Geom. Anal. 7 (1997).

[59] H.M. Soner, Ginzburg-Landau equation and motion by mean curvature. II. Development of the initial interface, J. Geom. Anal. 7 (1997).

[60] H.M. Soner, Convergence of the phase-field equations to the Mullins-Sekerka problem with kinetic undercooling [97d:80007], in: Fundamental Contributions to the Continuum Theory of Evolving Phase Interfaces in Solids, Springer, Berlin, 1999, pp. $413-471$.

[61] F. Stacy, Physics of the Earth, 2nd ed., Wiley, New York, 1977.

[62] M. Struwe, Variational Methods, Applications to Nonlinear Partial Differential Equations and Hamiltonian Systems, Springer, Berlin, 1990.

[63] J.E. Taylor, J.W. Cahn, Linking anisotropic sharp and diffuse surface motion laws via gradient flows, J. Stat. Phys. 77 (1994) $183-197$.

[64] J.E. Taylor, J.W. Cahn, Diffuse interfaces with sharp corners and facets: phase field models with strongly anisotropic surfaces, Physica D 112 (1998) 381-411 (with an appendix by Jason Yunger). 